\title{
Tracking Control Of An Underactuated Unmanned Underwater Vehicle
}

\author{
Abhijit Baviskar, Matthew Feemster ${ }^{\dagger}$, Darren Dawson, and Bin Xian Member, IEEE
}

\begin{abstract}
In this paper, a robust position tracking controller is developed for an autonomous, underactuated, unmanned underwater vehicle (UUV). Specifically, the proposed controller forces the three dimensional (i.e., longitudinal, latitudinal, and depth) position tracking error to within an arbitrarily small region about zero (i.e., global exponential uniformly ultimately bounded tracking) through the utilization of one translational and three orientation actuators. Therefore, the proposed methodology may hold special appeal to such underactuated applications as the omni-directional intelligent navigators (ODINs) by extending capabilities to include changes in vehicle depth. In addition, uncertainty in such parameters as vehicle inertia and hydrodynamic damping forces are compensated through a robust control structure. Simulation results are provided to illustrate performance and details of the controller.
\end{abstract}

\section{INTRODUCTION}

$\mathbf{T}$ HE autonomous underactuated vehicle control problem offers the challenging dilemma of developing a suitable control strategy that can simultaneously achieve a desired objective (position and/or orientation tracking/regulation) while utilizing a lower number of control inputs than degrees of freedom. For instance, a fully actuated vehicle would be equipped with three translational force actuators and three rotational torque actuators where any actuator can independently translate/rotate the vehicle along/around its respective axis. However, the weight of an autonomous vehicle is an obvious concern when considered in the context of aerial autonomous vehicles. Underwater vehicles are also plagued with the similar considerations as the neutral buoyancy depth will be affected by the overall weight and displacement of the vehicle.

Therefore, more advanced controllers are currently being investigated so that the tracking objective can be achieved in a satisfactory manner with a reduced number of actuators. For example, Behal et al. [2] developed a nonlinear controller of the kinematic model of an underactuated space craft that guaranteed uniform, ultimately bounded tracking provided that initial errors were selected sufficiently small. The control structure of [2] was motivated by a Lyapunov dynamic oscillator that originated in the area of wheeled

This work is supported in part by the U.S. NSF Grants DMI-9457967, ONR Grant N00014-99-1-0589, a DOC Grant, an ARO Automotive Center Grant, and ONR Grant N00014-03-WE20156.

Abhijit Baviskar and Darren Dawson are with Clemson University, Matthew Feemster is with the U.S. Naval Academy, and Bin Xian is with Duke University

†Corresponding Author: Phone/Fax: (410) 293-6139/(410) 293-2215, Email: feemster@usna.edu mobile robotics from [4]. Do et al. [5] presented an outputfeedback controller that guarantees global asymptotic stabilization tracking of an underactuated vehicle that operates at a constant depth (omni-directional intelligent navigator). In [14], a task-space tracking control approach for a redundant robot manipulator based on quaternion feedback was developed and is applied to an autonomous underwater vehicle by Xian et al. For further literature concerning the underactuated control problem please refer to [8], [11], [12], and [13] and the references within.

Recently, Aguiar and Hespanha [1] utilized an innovative approach to the two and three dimensional position tracking problem for underactuated autonomous vehicles. Specifically, a position tracking controller was developed that achieved global stability and exponential convergence of the position tracking error to within a neighborhood about zero which can be made arbitrarily small. Two distinctive techniques were employed that distinguished [1] from some of the previous work. First, a slightly modified expression for the dynamics of the rotation matrix was utilized throughout the control development which represents a shift from previous work. Second, Aguiar and Hespanha [1] augmented the filtered tracking error signal with a constant design vector. At first glance, this addition of a constant vector within a tracking error signal seems counterproductive; however, it is this additional design constant which facilitates the Lyapunov-based control synthesis. Unfortunately, the control development requires an input related matrix to be full rank. As mentioned in [1], it is not clear whether this full rank condition is always satisfied.

These two distinct approaches coupled with our previous research efforts in similar underactuated applications prompted us to investigate the possibility of relaxing the full rank condition for the tracking control problem. That is, the objective of our research is the development of a position tracking controller for an underactuated autonomous vehicle equipped with one translational actuator and three rotational actuators. To achieve this objective, the control design must be crafted in such a manner such that rotational torque can be "transmitted" to the translational system via coupling terms in order to influence position tracking. In addition, the controller must account for the fact that uncertainty exists within select parameter values. Therefore, a robust structure will be embedded within the control design. A stability analysis of the resulting tracking error signals demonstrates that the developed control strategy drives the position tracking error signal into a neighborhood about 
zero with an arbitrarily small radius set through the adjustment of design parameters. The approach of the proposed controller follows closely to that of [1]; however, the full rank condition set forth is [1] has been removed through a modified translational velocity error signal and through careful crafting of the error systems based on the stability analysis.

The paper is organized in the following manner. In Section II, the model of an underactuated UUV is presented. In Section III, the design objective of position tracking including the constraints of underactuation and parameter uncertainty is discussed. The controller development and subsequent stability analysis is presented in Section IV. Conclusions and topics for further investigation are presented in Section VI.

\section{SySTEM MODEL}

The kinematics of the UUV attitude can be represented by the unit quaternion [9] $\mathbf{q}(t)=\left[\begin{array}{ll}q_{o}(t) & q_{v}^{T}(t)\end{array}\right] \in \Re^{4}$, which describes the orientation of an orthogonal coordinate frame $\mathcal{B}$ attached to the UUV center of mass with respect to an inertial reference frame $\mathcal{I}$ expressed in $\mathcal{I}$ and is governed by the following differential equation [3]

$$
\begin{aligned}
& \dot{q}_{v}=\frac{1}{2}\left(q_{v} \times \omega+q_{o} \omega\right) \\
& \dot{q}_{o}=-\frac{1}{2} q_{v}^{T} \omega
\end{aligned}
$$

where $\omega(t) \in \Re^{3}$ is the angular velocity of $\mathcal{B}$ with respect to $\mathcal{I}$ expressed in $\mathcal{B}$.The rotation matrix that translates vehicle coordinates into inertial coordinates is denoted by $R(\mathbf{q}) \in$ $\Re^{3 \times 3}$ and is calculated from the following formula [10]

$$
R(\mathbf{q})=\left(q_{o}^{2}-q_{v}^{T} q_{v}\right) I_{3}+2 q_{v} q_{v}^{T}-2 q_{o} S\left(q_{v}\right)
$$

where $I_{3} \in \Re^{3 \times 3}$ is the $3 \times 3$ identity matrix, and $S(\cdot) \in \Re^{3 \times 3}$ is a skew-symmetric matrix with the following mapping characteristics

$$
S(\xi)=\left[\begin{array}{ccc}
0 & -\xi_{3} & \xi_{2} \\
\xi_{3} & 0 & -\xi_{1} \\
-\xi_{2} & \xi_{1} & 0
\end{array}\right] \quad \forall \xi \in \Re^{3}
$$

The translational kinematic relationship for the UUV is given by the following expression [7]

$$
\dot{p}=R v
$$

where $p(t) \in \Re^{3}$ represents the position of the origin of $\mathcal{B}$ expressed in $\mathcal{I}, v(t) \in \Re^{3}$ denotes the translational velocity of the UUV with respect to $\mathcal{I}$ expressed in $\mathcal{B}$. The translational and rotational equations of motion for the UUV are given by the following [1]

$$
\begin{aligned}
& M \dot{v}=-S(\omega) M v-h(v) v+B_{1} u_{1} \\
& J \dot{w}=-S(v) M v-S(\omega) J \omega-g(\omega) \omega+B_{2} u_{2}
\end{aligned}
$$

where $M, J \in \Re^{3 \times 3}$ denote the positive definite, constant mass and inertia matrices, respectively, $h(v) \in \Re^{3 \times 3}$ and $g(\omega) \in \Re^{3 \times 3}$ capture the linear and quadratic hydrodynamic damping interactions, $B_{1}=\left[\begin{array}{ccc}1 & 0 & 0\end{array}\right]^{T} \in \Re^{3}$, $u_{1}(t) \in \Re^{1}$ represents the translational force input, $B_{2}=$ $I_{3} \in \Re^{3 \times 3}$, and $u_{2} \in \Re^{3}$ denotes the rotation vector input.

Remark 1: The following properties of the rotation matrix for $R(\mathbf{q})$ and the skew-symmetric matrix $S(\cdot)$ will be utilized in the subsequent control development [1]

$$
\begin{array}{ll}
\text { I) } & \dot{R}=R S(\omega) \\
\text { II) } & R^{T} R=I_{3} \\
\text { III) } & S^{T}(y)=-S(y) \quad \forall y \in \Re^{3}
\end{array}
$$

As previously mentioned, Property I of (6) represents a slight shift in the structure of the dynamics for $R(\mathbf{q})$ [1] as compared to previous work.

\section{PROBLEM FORMULATION}

Our main objective is to design the translational force input $u_{1}(t)$ and the rotational torque input $u_{2}(t)$ such that $p(t)$ tracks a sufficiently smooth desired position trajectory $p_{d}(t) \in \Re^{3}$ (i.e., $p_{d}(t), \dot{p}_{d}(t), \ddot{p}_{d}(t), \dddot{p}_{d}(t) \in \mathcal{L}_{\infty}$ ). The position tracking objective is complicated due to the fact that the UUV is equipped with only one translational actuator aligned along one axis of $\mathcal{B}$. Therefore, the translational control input must be designed in conjunction with the rotational torque input such that the vehicle tracks $p_{d}(t)$. To this end, the position tracking error signal $e_{p}(t) \in \Re^{3}$ is defined in the following manner

$$
e_{p}=R^{T}\left(p-p_{d}\right) .
$$

In addition to the underactuation constraint, the control design must compensate for uncertainty in parameter values of the inertia matrix $J$, and the hydrodynamic damping coefficients in $h(v)$ and $g(\omega)$. In lieu of a dynamic estimation approach, a constant parameter estimate will be utilized in a feedforward term with the resulting parameter mismatch effects compensated for through the injection of a bounding function within the control input expressions for $u_{1}(t)$ and $u_{2}(t)$. The controller is designed under the assumption that the inertia matrix $M$ is known, and the translational position $p(t)$, the translational velocity $v(t)$, and the angular velocity $\omega(t)$ are measurable.

\section{Controller Design}

After taking the time derivative of (7) and substituting in the translational kinematics of (4), the open-loop tracking error dynamics for $e_{p}(t)$ are given by the following expression

$$
\begin{aligned}
\dot{e}_{p} & =-S(\omega) R^{T}\left(p-p_{d}\right)+v-R^{T} \dot{p}_{d} \\
& =-S(\omega) e_{p}+M^{-1} e_{v}+\left(M^{-1}-I_{3}\right) R^{T} \dot{p}_{d}
\end{aligned}
$$

where the properties of (6) have been utilized, the term $M^{-1} R^{T} \dot{p}_{d}$ has been added and subtracted to the right hand side of (8), and the translational velocity tracking error signal $e_{v}(t) \in \Re^{3}$ is defined in the following manner

$$
e_{v}=M v-R^{T} \dot{p}_{d}
$$


After taking the time derivative of $e_{v}(t)$ and substituting in the translational dynamics of (5), the open-loop linear velocity tracking error dynamics are given by the following

$$
\dot{e}_{v}=-S(\omega) e_{v}+\left(h(v) v-R^{T} \ddot{p}_{d}\right)+B_{1} u_{1} .
$$

where the definition of (9) has been utilized.

In order to simplify the control design, the filtered tracking error signal $r(t) \in \Re^{3}$ is defined in the following manner [1]

$$
r=e_{v}+\alpha e_{p}+\delta
$$

where $\alpha \in \Re^{1}$ denotes a positive, scalar constant, $\delta=$ $\left[\begin{array}{lll}\delta_{1} & 0 & 0\end{array}\right]^{T} \in \Re^{3}$, and $\delta_{1} \in \Re^{1}$ represents a positive design constant. The open-loop filtered tracking error dynamics for $r(t)$ are formulated by taking the time derivative of (11), substituting in the open-loop expressions of (8) and (10) and can be expressed in the following form

$$
\begin{aligned}
\dot{r}= & -S(\omega) r+S(\omega) \delta-h(v) v+B_{1} u_{1} \\
& +\alpha M^{-1} e_{v}-R^{T} \ddot{p}_{d}+\alpha\left(M^{-1}-I_{3}\right) R^{T} \dot{p}_{d}
\end{aligned}
$$

where the term $S(\omega) \delta \in \Re^{3}$ has been added and subtracted to the right hand side of (12). In order to facilitate the control design, it is assumed that $h(v) v$ in (12) can be linearly parameterized (LP) into the following form

$$
h(v) v=Y_{1}(v) \theta_{1}
$$

where $Y_{1}(v) \in \Re^{3 \times 6}$ represents a known regression matrix and $\theta_{1} \in \Re^{6}$ is the vector containing the unknown parameters. In addition, it is assumed that the parameter mismatch can be upperbounded in the following manner

$$
\left\|Y_{1}(v) \tilde{\theta}_{1}\right\| \leq \rho_{1}(\|v\|)
$$

where the parameter mismatch term is represented by $\tilde{\theta}_{1}=$ $\hat{\theta}_{1}-\theta_{1} \in \Re^{6}, \hat{\theta}_{1} \in \Re^{6}$ represents the "best" guess constant estimate of $\theta_{1}$, and $\rho_{1}(\|v(t)\|) \in \Re^{1}$ denotes a positive bounding function that is assumed to be non-decreasing in $\|v(t)\|$. The linearly parameterizing assumption of (13) should not be considered a limiting constraint on the structure of $h(v) v$ as the effects of parameter mismatch for the non-LP case could be easily incorporated into the robust control structure.

After substituting (13) into (12) for $h(v) v$, the open-loop filtered tracking error dynamics can be rewritten as follows

$$
\begin{aligned}
\dot{r}= & -S(\omega) r-Y_{1} \theta_{1}-M^{-1} e_{p} \\
& +\alpha M^{-1} e_{v}-R^{T} \ddot{p}_{d}+\alpha\left(M^{-1}-I_{3}\right) R^{T} \dot{p}_{d} \\
& +M^{-1} e_{p}+\left[B_{1} u_{1}-S(\delta) \omega\right]
\end{aligned}
$$

where the fact that $S(\omega) \delta=-S(\delta) \omega$ has been utilized and the term $M^{-1} e_{p}(t) \in \Re^{3}$ has been added and subtracted to the right hand side of (15). The bracketed term of (15) can be manipulated into the following form

$$
\left[B_{1} u_{1}-S(\delta) \omega\right]=B_{\mu} \mu
$$

where the auxiliary matrix $B_{\mu} \in \Re^{3 \times 3}$ and the auxiliary vector $\mu(t) \in \Re^{3}$ are defined by the following

$$
B_{\mu}=\left[\begin{array}{ccc}
1 & 0 & 0 \\
0 & 0 & \delta_{1} \\
0 & -\delta_{1} & 0
\end{array}\right] \quad \mu=\left[\begin{array}{lll}
u_{1} & \omega_{2} & \omega_{3}
\end{array}\right]^{T} \text {. }
$$

At this point, the scalar control input $u_{1}(t)$ is designed in the following manner

$$
u_{1}=\left[\begin{array}{lll}
1 & 0 & 0
\end{array}\right] \bar{u}_{1}+\omega_{1}
$$

where $\bar{u}_{1}(t) \in \Re^{3}$ represents an auxiliary control input vector. After substituting (18) into (16), the open-loop filtered tracking error dynamics for $r(t)$ can be expressed as follows

$$
\begin{aligned}
\dot{r}= & -S(\omega) r-Y_{1} \theta_{1}-M^{-1} e_{p} \\
& +\alpha M^{-1} e_{v}-R^{T} \ddot{p}_{d}+\alpha\left(M^{-1}-I_{3}\right) R^{T} \dot{p}_{d} \\
& +M^{-1} e_{p}+\left[B_{\mu} \bar{u}_{1}+B_{\mu} z\right]
\end{aligned}
$$

where the auxiliary vector signal $z(t) \in \Re^{3}$ is defined by the following

$$
z=\omega-B_{z} \bar{u}_{1}, \quad B_{z}=\left[\begin{array}{ccc}
0 & 0 & 0 \\
0 & 1 & 0 \\
0 & 0 & 1
\end{array}\right] .
$$

Based on the structure of (19), the auxiliary control input $\bar{u}_{1}(t)$ is designed in the following manner

$$
\begin{aligned}
\bar{u}_{1} & =B_{\mu}^{-1}\left[-\alpha M^{-1} e_{v}+R^{T} \ddot{p}_{d}-\alpha\left(M^{-1}-I_{3}\right) R^{T} \dot{p}_{d}\right. \\
& -M^{-1} e_{p}+Y_{1} \hat{\theta}_{1}-k_{r} r \\
& \left.-\left\{\frac{\rho_{1}^{2}\left(\|v\|_{s}\right) r}{\rho_{1}\left(\|v\|_{m}\right)\|r\|_{m}+\varepsilon_{1}}\right\}\right]
\end{aligned}
$$

where $k_{r} \in \Re^{1}$ denotes a positive scalar control gain, $\varepsilon_{1} \in$ $\Re^{1}$ represents a small positive constant, and the functions $\|\cdot\|_{s}$ and $\|\cdot\|_{m}$ are defined in the following manner

$$
\begin{aligned}
& \|y\|_{s}=\sqrt{y^{T} y+\sigma} \\
& \|y\|_{m}=\sqrt{y^{T} y+\sigma}-\sqrt{\sigma}=\left\|y_{s}\right\|-\sqrt{\sigma} \quad \forall y \in \Re^{3}
\end{aligned}
$$

where $\sigma \in \Re^{1}$ denotes a small positive scalar constant. After substituting (21) into the open-loop dynamics of (19), the closed-loop filtered tracking error dynamics for $r(t)$ are given by the following expression

$$
\begin{aligned}
\dot{r} & =-k_{r} r-S(\omega) r-M^{-1} e_{p}+B_{\mu} z \\
& +\left[Y_{1}(v) \tilde{\theta}_{1}-\frac{\rho_{1}^{2}\left(\|v\|_{s}\right) r}{\rho_{1}\left(\|v\|_{m}\right)\|r\|_{m}+\varepsilon_{1}}\right] .
\end{aligned}
$$

Remark 2: Based on the definitions of (22) and the non-decreasing characteristic of $\rho_{1}(\|v(t)\|)$, the following inequality will be used in the subsequent stability to bound the effects of the parameter mismatch term $Y_{1}(v) \tilde{\theta}_{1}$

$$
\rho_{1}\left(\|v\|_{s}\right) \geq \rho_{1}(\|v\|)>\rho_{1}\left(\|v\|_{m}\right) .
$$

Remark 3: The functions given in (22) are utilized in lieu of the standard Euclidean norm in $\bar{u}_{1}(t)$ so as to ensure that the time derivative of $\rho_{1}(\|v(t)\|)$ is well-defined when backstepping on the signal $z(t)$. That is, the time derivative 
of $\|\cdot\|_{s}$ and $\|\cdot\|_{m}$ can be calculated from the following expression

$$
\frac{d}{d t}\|y\|_{s}=\frac{d}{d t}\|y\|_{m}=\frac{y^{T} \dot{y}}{\sqrt{\left(y^{T} y+\sigma\right)}} \quad \forall y \in \Re^{3}
$$

The remainder of the control design involves the development of the torque input $u_{2}(t)$ such that the auxiliary variable $z(t)$ is regulated to zero. That is, if $z(t)=0$, then the auxiliary control input signal $\bar{u}_{1}(t)$ is injected into the filtered position tracking error dynamics (19) via the angular velocity vector $\omega(t)$ in order to promote position tracking.

After taking the time derivative of $z(t)$, multiplying both sides of the resulting expression by the unknown inertia matrix $J$, and substituting in the rotational dynamics of (5), the open-loop dynamics for $z(t)$ are given by the following expression

$$
\begin{aligned}
J \dot{z}= & -S(v) M v+\left[-S(\omega) J \omega-g(\omega) \omega-J B_{z} \dot{\bar{u}}_{1}\right] \\
& +B_{2} u_{2}
\end{aligned}
$$

and $\dot{\bar{u}}_{1}(t)$ expression is represented as

$$
\dot{\bar{u}}_{1}=B_{\mu}^{-1}\left[\phi_{a}+\phi_{b} \theta_{1}\right]
$$

where, $\phi_{a}(t) \in \Re^{3 \times 1}$ and $\phi_{b}(t) \in \Re^{3 \times 6}$ are two measurable regression matrices. At this point, the structure of $g(\omega) \omega$ is assumed to be of a form to allow the bracketed term of (26) to be linearly parameterized into the following form

$$
Y_{2}(p, \omega, v) \theta_{2}=-S(\omega) J \omega-g(\omega) \omega-J B_{z} \dot{\bar{u}}_{1}
$$

where $Y_{2}(p, \omega, v) \in \Re^{3 \times 23}$ denotes a known regression vector and $\theta_{2} \in \Re^{23}$ represents the vector of unknown constants. In addition, it is also assumed that the following bounding relationship exists

$$
\left\|Y_{2}(p, v, \omega) \tilde{\theta}_{2}\right\| \leq \rho_{2}(\|\zeta\|)
$$

where $\zeta(t)=\left[\begin{array}{lll}p^{T} & v^{T} & \omega^{T}\end{array}\right] \in \Re^{9}$, the parameter mismatch term is represented by $\tilde{\theta}_{2}=\theta_{2}-\hat{\theta}_{2} \in \Re^{23}$, $\hat{\theta}_{2} \in \Re^{23}$ represents the "best" guess constant estimate of $\theta_{2}$, and $\rho_{2}(\|\zeta\|) \in \Re^{1}$ denotes a positive bounding function that is designed to be non-decreasing in $\|\zeta(t)\|$. Similarly if the structure of $g(\omega) \omega$ does not allow for (28), then the effects of the bracketed terms of (26) could be compensated for through the development of an appropriate bounding function.

After substituting (28) into (26), the open loop dynamics for $z(t)$ are given by the following expression

$$
J \dot{z}=-S(v) M v+Y_{2} \theta_{2}+B_{2} u_{2} .
$$

Based on the structure of (30) and the subsequent stability analysis, the control torque $u_{2}(t)$ is designed in the following manner

$$
\begin{aligned}
u_{2} & =B_{2}^{-1}\left[S(v) M v-Y_{2} \hat{\theta}_{2}-k_{z} z-B_{\mu}^{T} r\right. \\
& \left.-\frac{\rho_{2}^{2}\left(\|\zeta\|_{s}\right) z}{\rho_{2}\left(\|\zeta\|_{m}\right)\|z\|_{m}+\varepsilon_{2}}\right]
\end{aligned}
$$

where $k_{z} \in \Re^{1}$ is represents a positive, scalar control gain and $\varepsilon_{2}$ represents a small positive constant. After substituting (31) into (30), the closed-loop dynamics for $z(t)$ are given by the following

$$
J \dot{z}=-k_{z} z-B_{\mu}^{T} r+\left\{Y_{2} \tilde{\theta}_{2}-\frac{\rho_{2}^{2}\left(\|\zeta\|_{s}\right) z}{\rho_{2}\left(\|\zeta\|_{m}\right)\|z\|_{m}+\varepsilon_{2}}\right\} .
$$

Theorem 1: The translational force input $u_{1}(t)$ of (18) and the rotational torque input $u_{2}(t)$ of (31) ensure that the tracking error signal $e_{p}(t)$ is exponentially driven into an arbitrarily small neighborhood about zero in the sense that

$$
\left\|e_{p}(t)\right\| \leq V(0) \exp \left(-\frac{\lambda_{3}}{\lambda_{2}} t\right)+\varepsilon
$$

where $\lambda_{3}, \lambda_{2}, \varepsilon \in \Re^{1}$ are positive constants (explicitly defined in the subsequent proof) and $V(0) \in \Re^{1}$ represents the initial condition of the subsequent Lyapunov candidate and is explicitly calculated as

$V(0)=\frac{1}{2} r^{T}(0) r(0)+\frac{1}{2} z^{T}(0) J z(0)+\frac{1}{2} e_{p}^{T}(0) e_{p}(0)$.

Proof: In order to illustrate the tracking result of (33), the following non-negative scalar function, denoted by $V(t)$, is defined as follows

$$
V=\frac{1}{2} r^{T} r+\frac{1}{2} z^{T} J z+\frac{1}{2} e_{p}^{T} e_{p},
$$

and $V(t)$ can be upper and lower bounded by the following inequality

$$
\lambda_{1}\|\eta\|^{2} \leq V \leq \lambda_{2}\|\eta\|^{2}
$$

where $\eta=\left[\begin{array}{lll}r^{T} & z^{T} & e_{p}^{T}\end{array}\right]^{T} \in \Re^{9}$ and the constant parameters $\lambda_{1}, \lambda_{2} \in \Re^{1}$ are given by

$$
\lambda_{1}=\min \left\{\frac{1}{2}, \frac{1}{2} \lambda_{\min }(J)\right\}, \lambda_{2}=\max \left\{\frac{1}{2}, \frac{1}{2} \lambda_{\max }(J)\right\}
$$

where $\lambda_{\min }\{J\}$ denotes the minimum eigenvalue of the matrix $J$. After taking the time derivative of (35), substituting in the closed-loop dynamics of (23), (32), and (8), and cancelling common terms, the time derivative of $V(t)$ is given by the following

$$
\begin{aligned}
\dot{V} & =-k_{r}\|r\|^{2}-k_{z}\|z\|^{2}-e_{p}^{T} \alpha M^{-1} e_{p} \\
& +e_{p}^{T}\left\{-M^{-1} \delta+\left(M^{-1}-I_{3}\right) R^{T} \dot{p}_{d}\right\} \\
& +\left[r^{T} Y_{1} \tilde{\theta}_{1}-\frac{\rho_{1}^{2}\left(\|v\|_{s}\right)\|r\|^{2}}{\rho_{1}\left(\|v\|_{m}\right)\|r\|_{m}+\varepsilon_{1}}\right] . \\
& +\left[z^{T} Y_{2} \tilde{\theta}_{2}-\frac{\rho_{2}\left(\|\zeta\|_{s}\right)\|z\|^{2}}{\rho_{2}\left(\|\zeta\|_{m}\right)\|z\|_{m}+\varepsilon_{2}}\right]
\end{aligned}
$$

After utilizing (14), (29), and (24), $\dot{V}(t)$ of (38) can be upperbounded in the following manner

$$
\begin{aligned}
\dot{V} & \leq-k_{r}\|r\|^{2}-\left[\alpha \lambda_{\min }\left\{M^{-1}\right\}-\frac{\varepsilon_{3}^{2}}{2}\right]\left\|e_{p}\right\|^{2} \\
& -k_{z}\|z\|^{2}+\left(\varepsilon_{1}+\varepsilon_{2}+\frac{\eta_{0}^{2}}{2 \varepsilon_{3}}\right)
\end{aligned}
$$


where $\varepsilon_{3} \in \Re^{1}$ represents a positive constant and $\eta_{0} \in$ $\Re^{1}$ denotes a positive bounding constant that satisfies the following inequality

$$
\eta_{0}>\left\|-M^{-1} \delta+\left(M^{-1}-I_{3}\right) R^{T} \dot{p}_{d}\right\|
$$

If the control gain $\alpha$ is selected such that

$$
\alpha>\frac{\varepsilon_{3}}{2 \lambda_{\min }\left\{M^{-1}\right\}},
$$

then the time derivative of $V(t)$ can be rewritten as

$$
\dot{V} \leq-\lambda_{3}\|\eta\|^{2}+\varepsilon \leq-\frac{\lambda_{3}}{\lambda_{2}} V+\varepsilon
$$

where $\lambda_{3}=\min \left\{k_{r}, \quad k_{z}, \quad \alpha \lambda_{\min }\left\{M^{-1}\right\}-\frac{\varepsilon_{3}^{2}}{2}\right\} \in$ $\Re^{1}, \varepsilon=\left(\varepsilon_{1}+\varepsilon_{2}+\frac{\eta_{0}^{2}}{2 \varepsilon_{3}}\right) \in \Re^{1}$, and (36) has been utilized. From (42), it can be observed that $V(t)$ is driven exponentially into a ball with radius $\varepsilon$. Standard linear arguments can then be applied to (42) to obtain (33) in the sense that

$$
\left\|e_{p}(t)\right\| \leq V(t) \leq V(0) \exp \left(-\frac{\lambda_{3}}{\lambda_{2}} t\right)+\varepsilon
$$

Standard signal tracing can now be employed to illustrate that all signals remain bounded during closed-loop operation.

\section{Simulation Results}

In order to evaluate performance and to observe the details of implementation, the UUV system of (1) through (5) was simulated in MATLAB's Simulink software package. The explicit structure of the matrices of (5) are given as follows

$$
\begin{gathered}
M=\left[\begin{array}{ccc}
m_{1} & 0 & 0 \\
0 & m_{2} & 0 \\
0 & 0 & m_{3}
\end{array}\right], \quad J=\left[\begin{array}{ccc}
J_{1} & 0 & 0 \\
0 & J_{2} & 0 \\
0 & 0 & J_{3}
\end{array}\right] \\
h(v)=\left[\begin{array}{ccc}
h_{1}+h_{2}\left|v_{1}\right| & 0 & 0 \\
0 & h_{3}+h_{4}\left|v_{2}\right| & 0 \\
0 & 0 & h_{5}+h_{6}\left|v_{3}\right|
\end{array}\right], \\
g(\omega)=\left[\begin{array}{ccc}
g_{1}+g_{2}\left|\omega_{1}\right| \\
0 & g_{3}+g_{4}\left|\omega_{2}\right| & 0 \\
0 & 0 & g_{5}+g_{6}\left|\omega_{3}\right|
\end{array}\right]
\end{gathered}
$$

where the parameter values for the system were selected according to [6]. The initial attitude of the vehicle was adjusted such that the UUV's $x$-axis was aligned with the $x$-axis of the $\mathcal{I}$ frame by setting $\mathbf{q}(0)$ to the following

$$
\mathbf{q}(0)=\left[\begin{array}{llll}
1.0 & 0.0 & 0.0 & 0.0
\end{array}\right]^{T},
$$

and the initial position of the center of mass of the UUV was selected as $p(0)=\left[\begin{array}{lll}-5.0 & -5.0 & -5.0\end{array}\right]^{T}(m)$. To illustrate the position tracking capabilities of the proposed controller, the following soft start trajectory was selected

$$
p_{d}(t)=\left[\begin{array}{c}
20.0\left(1.0-e^{\left(-0.05\left(t-\tau_{x}\right)^{3}\right)}\right) \\
20.0\left(1.0-e^{\left(-0.05\left(t-\tau_{y}\right)^{3}\right)}\right) \\
20.0\left(1.0-e^{\left(-0.05\left(t-\tau_{z}\right)^{3}\right)}\right)
\end{array}\right](m)
$$

where the constants $\tau_{x}, \tau_{y}$, and $\tau_{z} \in \Re^{1}$ were selected as follows

$$
\tau_{x}=0.0 \quad \tau_{y}=10.0 \quad \tau_{z}=20.0 \quad(\mathrm{sec}) .
$$

(Note: if the argument $\left(t-\tau_{i}\right)$ in the exponential term of (48) evaluates to less than zero, then the desired position for that element is set to zero).

The parameter estimate vectors $\hat{\theta}_{1} \in \Re^{6}$ and $\hat{\theta}_{2} \in \Re^{23}$ were set to $50 \%$ of their corresponding nominal values of. The following control gains were utilized for the simulation

$$
\begin{array}{llll}
k_{r}=10.0 & k_{z}=10.0 & \alpha=150.0 & \delta_{1}=0.1 \\
\sigma=0.01 & \varepsilon_{1}=1.0 & \varepsilon_{2}=1.0
\end{array}
$$

The structure of the bounding functions $\rho_{1}(\|v\|)$ and $\rho_{2}(\|\zeta\|)$ of (14) and (29), respectively, were selected as follows

$$
\rho_{1}=\sqrt{A\left(\|v\|^{2}+\|v\|^{4}\right)+B} \quad \rho_{2}=\sqrt{C\left\|Y_{2}(\zeta) \phi\right\|^{2}}
$$

where $\phi=\left[\begin{array}{llll}1 & 1 & \ldots & 1\end{array}\right]^{T} \in \Re^{23}$. In order to make an educated guess on the values the bounding constants $A$, $B$, and $C, \in \Re^{1}$, we will assume that a range for each parameter value is known. That is, the vectors $\bar{\theta}_{1} \in \Re^{6}$ and $\bar{\theta}_{2} \in \Re^{23}$ represent the upperbound values for $\theta_{1}$ and $\theta_{2}$. For our simulation we will assume that we know the system parameters to within $500 \%$ of their nominal value (i.e., $\bar{\theta}_{1}=5 \theta_{1}$ and $\bar{\theta}_{2}=5 \theta_{2}$ ); therefore, $A$ and $C$ are selected as the maximum element of $\bar{\theta}_{1}$ and $\bar{\theta}_{2}$ as given by

$$
A=\max \left\{\bar{\theta}_{1}\right\} \quad B=0.1 \quad C=\max \left\{\bar{\theta}_{2}\right\} .
$$

Figure 1 illustrates the UUV's three dimensional trajectory to the target coordinate. Figure 2 shows the position tracking error along each axis, and Figure 3 provides the translational and rotational control force/torques inputs.

The desired trajectory of (48) and (49) was selected such that two 90 degree "turns" were required in order for the UUV to reach the target coordinate. One expects the proposed controller to align the translational actuator along a path so as to reduce the position error signal. Since we do not control the orientation of the UUV directly, it is interesting to observe in what manner the UUV will orient itself during the cornering maneuver. That is, will the UUV align itself in such a manner as to "push" (a positive $u_{1}(t)$ ) or "pull" (a negative $u_{1}(t)$ ) the UUV along the correct trajectory. As seen from Figures 1 and 3, the UUV oriented itself such that a positive thrust $u_{1}(t)$ was commanded for both changes in direction and the initial quaternion vector of (47). 


\section{CONCLUSION}

In this paper, a controller has been presented that provides three dimensional position tracking (i.e., longitudinal, latitudinal, and depth) of an unmanned underwater vehicle with only one translational actuator and three axial torque actuators. The robust control structure also compensates for parameter uncertainty in the vehicle inertia and hydrodynamic damping coefficients. The presented controller extends upon the work of [1] by removing the full rank on the input matrix condition by utilizing a modified translational velocity error signal and by carefully crafting the tracking error systems from the stability analysis. Future work will include the investigation of incorporating flywheels into the underactuated UUV application to serve as both energy source and torque actuator.

\section{REFERENCES}

[1] Aguiar, A. and Joao Hespanha, "Position Tracking of Underactuated Vehicles," Proceedings of the American Control Conference, pp. 1988-1993, Denver, CO, 2003.

[2] Behal, A., D. Dawson, E. Zergeroglu, and Y. Fang, "Nonlinear Tracking Control of an Underactuated Spacecraft," AIAA Journal of Guidance, Control, and Dynamics, Vol. 25, No. 5, pp. 979-985.

[3] Costic, B., M. de Queiroz, and D. Dawson, "Energy Management And Attitude Control Strategies Using Flywheels," Proceedings of the IEEE Conference Decision and Control, Orlando, FL, Dec. 2001, pp. 3435-3440.

[4] Dixon, W., D. Dawson, E. Zergeroglu, and F. Zhang, "Robust Tracking and Regulation for Mobile Robots," International Journal of Robust and Nonlinear Control: Special Issue on Control of Underactuated Nonlinear Systems, Vol. 10, 2000, pp. 199-216.

[5] Do, K., Z. Jiang, J. Pan, and H. Nijmeijer, "A Global OutputFeedback Controller For Stabilization and Tracking of Underactuated ODIN: A Spherical Underwater Vehicle," Automatica, Vol. 40, pp. 117-124.

[6] Fjellstad, O. and Thor I. Fossen, Quaternion Feedback Regulation of Underwater Vehicles, Proceeding IEEE Conference on Control Applications, Strathclyde University, Glasgow, 1994, pp. 857-862

[7] Fossen, T., Guidance and Control of Ocean Vehicles, Chichester, UK: John Wiley \& Sons, 1994.

[8] Godhavn, J., "Nonlinear Tracking of Underactuated Surface Vessels," Proceedings of the 35th Conference on Decision and Control, Kobe, Japan, Dec. 1996, pp. 975-980.

[9] Huches, P.C., Spacecraft Attitude Dynamics, New York, NY: Wiley, 1994.

[10] Lizarralde, F. and J.T. Wen, "Attitude Control Without Angular Velocity Measurement: A Passivity Approach," IEEE Transactions on Automatic Control, Vol. 41, No. 3, pp. 468-472.

[11] Pettersen, K. and H. Nijmeijer, "Global Practical Stabilization and Tracking for an Underactuated Ship - A Combined Averaging and Backstepping Approach," Proceedings of the IFAC Conference on System Structure and Control, Nantes, France, July 1998, pp. 59-64.

[12] Pettersen, R., and O. Egeland, "Exponential Stabilization of an Underactuated Surface Vessel," Modeling, Identification and Control, Vol. 18, No. 3, pp. 239.

[13] Reyhanoglu, M., A. Schaft, N. McClamroch, and I. Kolmanovsky, "Dynamics and Control of a Class of Underactuated Mechanical Systems," IEEE Transactions on Automatic Control, Vol. 44, No. 9, pp. 1663-1670, 1999.

[14] Xian, B., M.S. de Queiroz, D. Dawson, and I. Walker, "TaskSpace Tracking Control of Robot Manipulators via Quaternion Feedback," IEEE Transactions on Robotics and Automation, Vol. 20, No. 1,pp.160-167.

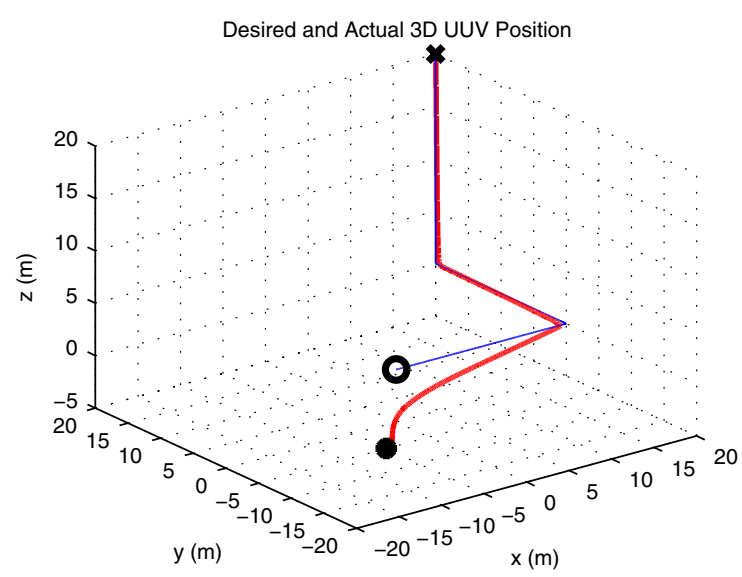

Fig. 1. 3D Position Trajectory

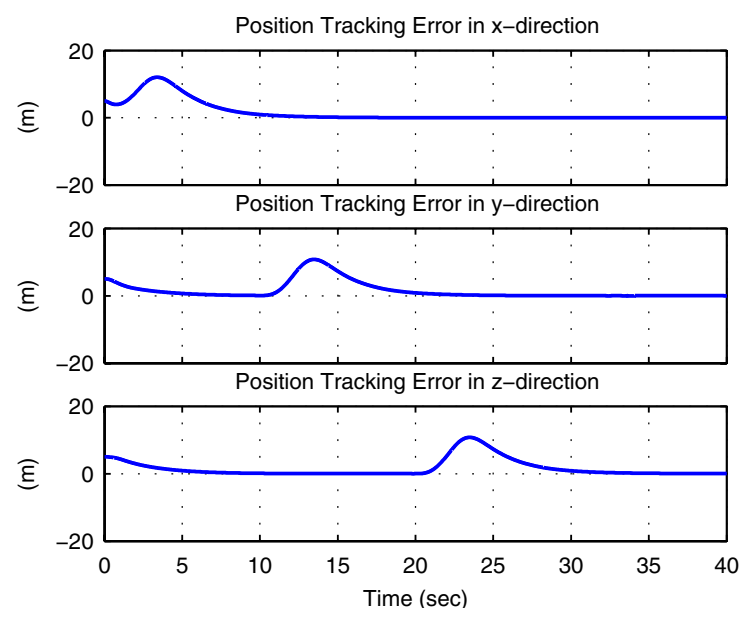

Fig. 2. Position Tracking Error Signals

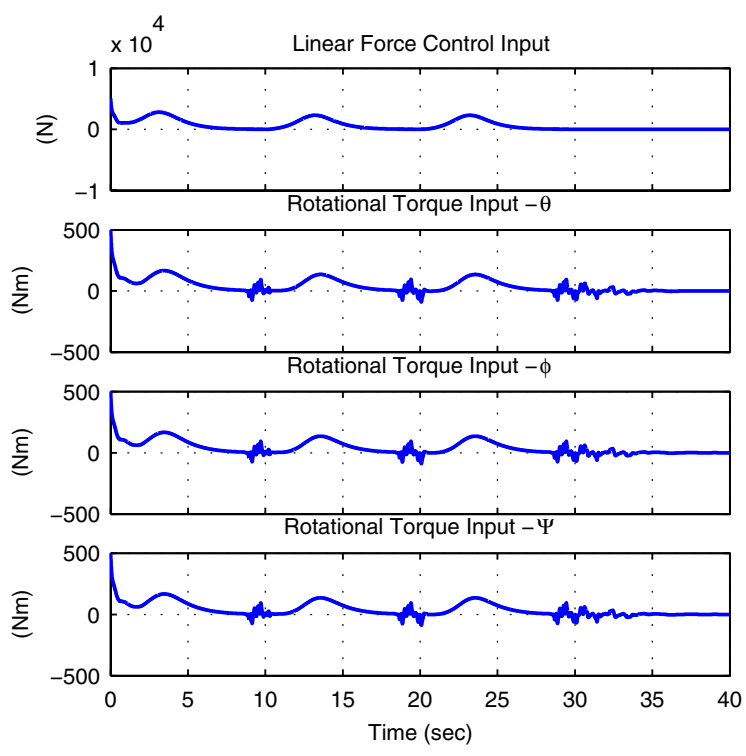

Fig. 3. Control Input Signals 